\begin{tabular}{|c|l|}
\hline Title & Sobolev inequalities with symmetry \\
\hline Author(s) & Cho, Yonggeun; Ozawa, Tohru \\
\hline Citation & Hokkaido University Preprint Series in Mathematics, 859, 1-8 \\
\hline Issue Date & 2007 \\
\hline DOI & 10.14943/84009 \\
\hline Doc URL & http://hdl.handle.net/2115/69668 \\
\hline Type & bulletin (article) \\
\hline File Information & pre859.pdf \\
\hline
\end{tabular}

Instructions for use 


\title{
SOBOLEV INEQUALITIES WITH SYMMETRY
}

\author{
YONGGEUN CHO AND TOHRU OZAWA
}

\begin{abstract}
In this paper we derive some Sobolev inequalities for radially symmetric functions in $\dot{H}^{s}$ with $\frac{1}{2}<s<\frac{n}{2}$. We show the end point case $s=\frac{1}{2}$ on the homogeneous Besov space $\dot{B}_{2,1}^{\frac{1}{2}}$. These results are extensions of the well-known Strauss' inequality [11]. Also non-radial extensions are given, which show that compact embeddings are possible in some $L^{p}$ spaces if a suitable angular regularity is imposed.
\end{abstract}

\section{INTRODUCTION}

In this paper we derive Sobolev inequalities with symmetry. We first consider several Sobolev inequalities for radially symmetric functions in $\dot{H}^{s}\left(\mathbb{R}^{n}\right)$ with $\frac{1}{2}<s<\frac{n}{2}$. There is a sharp result by Sickel and Skrzypczak [8], although the argument below is much simpler and direct and a constant in the inequality in Proposition 1 below is given explicitly in terms of $s$.

\section{Definition 1.}

$$
\begin{aligned}
& \dot{H}_{\mathrm{rad}}^{s}=\left\{u \in \dot{H}^{s}\left(\mathbb{R}^{n}\right): u \text { is radially symmetric }\right\}, s \geq 0 . \\
& \dot{B}_{p, q, \text { rad }}^{s}=\left\{u \in \dot{B}_{p, q}^{s}\left(\mathbb{R}^{n}\right): u \text { is radially symmetric }\right\}, s \geq 0,1 \leq p, q \leq \infty .
\end{aligned}
$$

The inhomogeneous spaces of radially symmetric functions are defined by the same way with spaces $H^{s}$ and $B_{p, q}^{s}$.

Proposition 1. Let $n \geq 2$ and let $s$ satisfy $1 / 2<s<n / 2$.

Then

$$
\sup _{x \in \mathbb{R}^{n} \backslash\{0\}}|x|^{n / 2-s}|u(x)| \leq C(n, s)\left\|(-\Delta)^{s / 2} u\right\|_{L^{2}}
$$

for all $u \in \dot{H}_{\text {rad }}^{s}$, where

$$
C(n, s)=\left(\frac{\Gamma(2 s-1) \Gamma\left(\frac{n}{2}-s\right) \Gamma\left(\frac{n}{2}\right)}{2^{2 s} \pi^{n / 2} \Gamma(s)^{2} \Gamma\left(\frac{n}{2}-1+s\right)}\right)^{1 / 2}
$$

and $\Gamma$ is the gamma function.

Remark 1. For $s=1$ with $n \geq 3$, the inequality (1) reduces to Ni's inequality $[6,7]$.

Remark 2. The restriction $1 / 2<s<n / 2$ is necessary for $C(n, s)$ to be finite.

2000 Mathematics Subject Classification. Primary 46E35; Secondary 42C15.

Keywords and phrases. Sobolev inequality, function space with radial symmetry, angular regularity. 
Remark 3. The inequality (1) fails for $s=n / 2$. Indeed, $u(x)=\mathcal{F}^{-1}\left(\frac{1}{(1+|\xi|)^{n}(1+\log (1+|\xi|))}\right)$ satisfies $u \in H_{\text {rad }}^{n / 2}$, and $u \notin L^{\infty}$ where $\mathcal{F}$ is the Fourier transform [12] and $\mathcal{F}^{-1}$ is its inverse.

Remark 4. The inequality (1) fails if $0 \leq s<1 / 2$ and $n \geq 3$. Indeed, $u=$ $\mathcal{F}^{-1}\left(J_{\frac{n}{2}-1}(|\xi|)|\xi|^{-n / 2}\right)$ satisfies $u \in \dot{H}_{\text {rad }}^{s}$ and $u(x)=\infty$ for all $x \in S^{n-1}$, where we note that $u \in \dot{H}_{\text {rad }}^{s}$ if and only if $1-n / 2<s<1 / 2$, since

$$
\left\|(-\Delta)^{s / 2} u\right\|_{L^{2}}^{2}=c_{n} \int_{0}^{\infty}\left|J_{\frac{n}{2}-1}(\rho)\right|^{2} \rho^{2 s-1} d \rho
$$

and that by the asymptotic behavior of Bessel function (10)

$$
u(x)=\int_{0}^{\infty}\left|J_{\frac{n}{2}-1}(\rho)\right|^{2} d \rho=\infty, \quad x \in S^{n-1} .
$$

See also the proof of Proposition 1 below.

In the endpoint case $s=1 / 2$, we have the following propostion.

Proposition 2. Let $n \geq 2$. Then there exists a constant $C$ such that

$$
\sup _{x \in \mathbb{R}^{n} \backslash\{0\}}|x|^{(n-1) / 2}|u(x)| \leq C\|u\|_{\dot{B}_{2,1}^{1 / 2}}
$$

for all $u \in \dot{B}_{2,1, \mathrm{rad}}^{1 / 2}$.

Remark 5. The inhomogeneous version of (2) has been given in [8] whose proof is based on the atomic decomposition.

Proposition 3. Let $n \geq 2$ and let s satisfy $1 / 2 \leq s<1$. Then there exists $C$ such that for all $u \in H_{\mathrm{rad}}^{1}$

$$
\sup _{x \in \mathbb{R}^{n} \backslash\{0\}}|x|^{n / 2-s}|u(x)| \leq C(n, s)\|u\|_{L^{2}}^{1-s}\|\nabla u\|_{L^{2}}^{s} .
$$

Remark 6. For $s=1 / 2$, the inequality (3) reduces to Strauss' inequality [11].

Remark 7. For $s=0$, the inequality (3) holds for nonincreasing functions in $|x|$ [2]. For $s=1$, the inequality (3) holds for $n \geq 3$ and fails for $n=2$. See Proposition 1 and Remark 2.

Now we extend the results above on radial functions to the non-radial functions with additional angular regularity. For details, let us define function spaces $H_{\omega}^{s, m}$ and $B_{2,1, \omega}^{s, m}$, $s \geq 0, m \geq 0$ as follows. 
Definition 2.

$$
\begin{aligned}
& H_{\omega}^{s, m}=\left\{u \in H^{s}:\|u\|_{H_{\omega}^{s, m}} \equiv\left\|\left(1-\Delta_{\omega}\right)^{\frac{m}{2}} u\right\|_{H^{s}}<\infty\right\}, \\
& B_{2,1, \omega}^{s, m}=\left\{u \in B_{2,1}^{\frac{1}{2}}:\|u\|_{B_{2,1, \omega}^{s, m}}^{2} \equiv\left\|\left(1-\Delta_{\omega}\right)^{\frac{m}{2}} u\right\|_{B_{2,1}^{s}}<\infty\right\},
\end{aligned}
$$

where $\Delta_{\omega}$ is the Laplace-Beltrami operator on $S^{n-1}$.

The homogeneous spaces $\dot{H}_{\omega}^{s, m}$ and $\dot{B}_{2,1, \omega}^{s, m}$ is similarly defined by the definition of $\dot{H}^{s}$ and $\dot{B}_{2,1}^{s}$. Then we have the following.

Proposition 4. (1) If $1 / 2<s<n / 2$ and $m>n-1-s$, then there exists a constant $C$ such that for any $u \in H_{\omega}^{s, m}$

$$
\sup _{\mathbb{R}^{n} \backslash\{0\}}|x|^{n / 2-s}|u(x)| \leq C\|u\|_{\dot{H}_{\omega}^{s, m}} .
$$

(2) If $m>n-\frac{3}{2}$, then there exists a constant $C$ such that for any $u \in B_{2,1, \omega}^{\frac{1}{2}, m}$

$$
\sup _{\mathbb{R}^{n} \backslash\{0\}}|x|^{(n-1) / 2}|u(x)| \leq C\|u\|_{\dot{B}_{2,1, \omega}^{\frac{1}{2}, m}} .
$$

Remark 8. $\quad H_{\omega}^{s, m}$ and $B_{2,1, \omega}^{\frac{1}{2}, m}$ are closed subspaces of $H^{s}$ and $B_{2,1}^{s}$, respectively and they contain $H_{\text {rad }}^{s}$ and $B_{2,1, \text { rad }}^{\frac{1}{2}}$ naturally, respectively. We can identify the spaces $H^{s}$ with $\left(1-\Delta_{\omega}\right)^{m / 2} H_{\omega}^{s, m}$ and also $B_{2,1}^{\frac{1}{2}}$ with $\left(1-\Delta_{\omega}\right)^{m / 2} B_{2,1, \omega}^{\frac{1}{2}, m}$.

Remark 9. $H_{\omega}^{s, m}$ is a Hilbert space with the same inner product as $L^{2}$ space and its dual space is given by $H_{\omega}^{-s,-m}$.

From the decay at infinity we deduce compact embeddings of $H_{\omega}^{s, m}$ and $B_{2,1, \omega}^{\frac{1}{2}, m}$ into some $L^{p}$ spaces as follows. See $[2,3,4,8,9]$ for the radial case.

Corollary 1. The embedding $H_{\omega}^{s, m} \hookrightarrow L^{p}$ is compact for $1 / 2<s<n / 2, m>n-1-s$ and $2<p<2 n /(n-2 s)$.

Corollary 2. The embedding $B_{2,1, \omega}^{\frac{1}{2}, m} \hookrightarrow L^{p}$ is compact for $m>n-3 / 2$ and $2<p<$ $2 n /(n-1)$.

\section{Proofs}

2.1. Proof of Proposition 1. We use the following Fourier representation for radially symmetric functions as

$$
u(x)=|x|^{1-\frac{n}{2}} \int_{0}^{\infty} J_{\frac{n}{2}-1}(|x| \rho) \widehat{u}(\rho) \rho^{\frac{n}{2}} d \rho,
$$

where $J_{\nu}$ is the Bessel function of order $\nu, \widehat{u}$ is the Fourier transform normalized as

$$
\widehat{u}(\xi)=(2 \pi)^{-n / 2} \int e^{-i x \cdot \xi} u(x) d x,
$$


and we have identified radially symmetric functions on $\mathbb{R}^{n}$ with the corresponding functions on $(0, \infty)$.

By the Cauchy-Schwarz inequality and the Plancherel formula, we have

$$
\begin{aligned}
& |x|^{\frac{n}{2}-s}|u(x)| \\
& \leq|x|^{1-s}\left(\int_{0}^{\infty}\left|J_{\frac{n}{2}-1}(|x| \rho)\right|^{2} \rho^{1-2 s} d \rho\right)^{1 / 2}\left(\int_{0}^{\infty}|\widehat{u}(\rho)|^{2} \rho^{2 s+n-1} d \rho\right)^{1 / 2} \\
& =\left(\int_{0}^{\infty}\left|J_{\frac{n}{2}-1}(\rho)\right|^{2} \rho^{1-2 s} d \rho\right)^{1 / 2}\left(\frac{\Gamma\left(\frac{n}{2}\right)}{2 \pi^{n / 2}} \int|\xi|^{2 s}|\widehat{u}(\xi)|^{2} d \xi\right)^{1 / 2} \\
& =C(n, s)\left\|(-\Delta)^{s / 2} u\right\|_{L^{2}},
\end{aligned}
$$

as required.

2.2. Proof of Proposition 2. We use the following estimates on Bessel functions:

$$
\begin{aligned}
& \sup _{r \geq 0}\left|J_{\frac{n}{2}-1}(r)\right| \leq 1 . \\
& \sup _{r \geq 0} r^{1 / 2}\left|J_{\frac{n}{2}-1}(r)\right| \leq C .
\end{aligned}
$$

The first inequality (8) follows from the integral representation (see [13])

$$
\begin{aligned}
& J_{\frac{n}{2}-1}(r)^{2}=\frac{2}{\pi} \int_{0}^{\pi / 2} J_{n-2}(2 r \cos \theta) d \theta, \\
& J_{m}(t)=\frac{1}{2 \pi} \int_{0}^{2 \pi} \cos (m \theta-t \sin \theta) d \theta, m \in \mathbb{Z} .
\end{aligned}
$$

The second inequality (9) follows from the first and the well-known asymptotics (see [10])

$$
J_{\nu}(r) \sim \sqrt{\frac{2}{\pi r}} \cos \left(r-\frac{(2 \nu+1) \pi}{4}\right) \quad \text { as } \quad r \rightarrow \infty .
$$

We apply the Littlewood-Paley decomposition $\left\{\varphi_{j}\right\}_{j \in \mathbb{Z}}$ on $\mathbb{R}^{n} \backslash\{0\}$ to (4) to obtain

$$
u(x)=|x|^{1-\frac{n}{2}} \sum_{j \in \mathbb{Z}} \int_{0}^{\infty} J_{\frac{n}{2}-1}(|x| \rho) \psi_{j}(\rho) \varphi_{j}(\rho) \widehat{u}(\rho) \rho^{\frac{n}{2}} d \rho,
$$

where $\psi_{j}=\varphi_{j-1}+\varphi_{j}+\varphi_{j+1}$ and $\operatorname{supp} \varphi_{j} \subset\left\{2^{j-1} \leq \rho \leq 2^{j+1}\right\}$.

As in (7), we have

$$
\begin{aligned}
& |x|^{(n-1) / 2}|u(x)| \\
& \leq|x|^{1 / 2} \sup _{j \in \mathbb{Z}}\left(\int_{0}^{\infty}\left|J_{\frac{n}{2}-1}(|x| \rho)\right|^{2} \psi_{j}(\rho)^{2} d \rho\right)^{1 / 2} \\
& \cdot \sum_{j \in \mathbb{Z}}\left(\int_{0}^{\infty}\left|\varphi_{j}(\rho) \widehat{u}(\rho)\right|^{2} \rho^{n} d \rho\right)^{1 / 2} .
\end{aligned}
$$


By (9), we estimate

$$
\begin{aligned}
& |x|^{1 / 2} \sup _{j \in \mathbb{Z}}\left(\int_{0}^{\infty}\left|J_{\frac{n}{2}-1}(|x| \rho)\right|^{2} \psi_{j}(\rho)^{2} d \rho\right)^{1 / 2} \\
& \leq C \sup _{j \in \mathbb{Z}}\left(\int_{0}^{\infty} \frac{1}{\rho} \psi_{j}(\rho)^{2} d \rho\right)^{1 / 2} \\
& \leq C \sup _{j \in \mathbb{Z}}\left(\int_{2^{j-2}}^{2^{j+2}} \frac{1}{\rho} d \rho\right)^{1 / 2} \leq C .
\end{aligned}
$$

This proves (2) since

$$
\sum_{j \in \mathbb{Z}}\left(\int_{0}^{\infty}\left|\varphi_{j}(\rho) \widehat{u}(\rho)\right|^{2} \rho^{n} d \rho\right)^{1 / 2}
$$

is equivalent to the seminorm on $\dot{B}_{2,1, \mathrm{rad}}^{1 / 2}$.

2.3. Proof of Proposition 3. If we use Cauchy-Schwartz inequality as in (7), we have for any $M>0$

$$
\begin{aligned}
& |x|^{n / 2-s}|u(x)| \\
& \leq|x|^{1-s}\left(\int_{0}^{M|x|}\left|J_{\frac{n}{2}-1}(|x| \rho)\right|^{2} \rho d \rho\right)^{1 / 2}\left(\int_{0}^{M|x|}|\widehat{u}(\rho)|^{2} \rho^{n-1} d \rho\right)^{1 / 2} \\
& +|x|^{1-s}\left(\int_{M|x|}^{\infty}\left|J_{\frac{n}{2}-1}(|x| \rho)\right|^{2} \rho^{-1} d \rho\right)^{1 / 2}\left(\int_{M|x|}^{\infty}|\widehat{u}(\rho)|^{2} \rho^{n+1} d \rho\right)^{1 / 2} \\
& \leq|x|^{-s}\left(\int_{0}^{M}\left|J_{\frac{n}{2}-1}(r)\right|^{2} r d r\right)^{1 / 2}\|u\|_{L^{2}} \\
& +|x|^{1-s}\left(\int_{M}^{\infty}\left|J_{\frac{n}{2}-1}(r)\right|^{2} r^{-1} d r\right)^{1 / 2}\|\nabla u\|_{L^{2}} .
\end{aligned}
$$

From (8) and (9) we deduce that $\sup _{r \geq 0}\left|r^{1-s} J_{\frac{n}{2}-1}(r)\right| \leq C$ for any $\frac{1}{2} \leq s<1$. Hence we have for any $M>0$

$$
\begin{aligned}
& |x|^{n / 2-s}|u(x)| \\
& \leq|x|^{-s}\left(\int_{0}^{M}\left|J_{\frac{n}{2}-1}(r)\right|^{2} r d r\right)^{\frac{1}{2}}\|u\|_{L^{2}} \\
& +|x|^{1-s}\left(\int_{M}^{\infty}\left|J_{\frac{n}{2}-1}(r)\right|^{2} r^{-1} d r\right)^{\frac{1}{2}}\|\nabla u\|_{L^{2}} \\
& \leq C|x|^{-s} M^{s}\|u\|_{L^{2}}+|x|^{1-s} M^{-(1-s)}\|\nabla u\|_{L^{2}} .
\end{aligned}
$$

The minimization of the RHS of the last inequality with respect to $M$ yields Proposition 3. 
2.4. Proofs of Proposition 4 and Corollaries. The proof for (4) follows from the one of Proposition 1 and the spherical harmonic expansion of functions in $H_{\omega}^{s, m}[5,10]$. In fact, if we write $u(r \omega)=\sum_{k \geq 0} \sum_{1 \leq l \leq d(k)} f_{k, l}(r) Y_{k, l}(\omega)$, where $d(k)$ is the dimension of space of spherical harmonic functions of degree $k$ and

$$
d(k) \leq C k^{n-2} \text { for large } k .
$$

Then we have

$$
|x|^{\frac{n}{2}-s} u(|x| \omega)=c_{n} \sum_{k, l}|x|^{1-s} \int_{0}^{\infty} J_{\nu(k)}(|x| \rho) \rho^{\frac{n}{2}} g_{k, l}(\rho) d \rho Y_{k, l}(\omega),
$$

where $\omega \in S^{n-1}, \nu(k)=\frac{n+2 k-2}{2}$ and $\widehat{f_{k, l} Y_{k, l}}(\rho \omega)=g_{k, l}(\rho) Y_{k, l}(\omega)$. Here

$$
g_{k, l}(\rho)=c_{n, k} \int_{0}^{\infty} f_{k, l}(r) r^{n-1}(r \rho)^{-\frac{n-2}{2}} J_{\nu(k)}(r \rho) d r .
$$

The absolute value of $c_{n, k}$ is bounded by a constant depending only on $n$. See [10] for this.

Using the Cauchy-Schwarz inequality as in (7), we have

$$
\begin{aligned}
& |x|^{\frac{n}{2}-s}|u(|x| \omega)| \\
& \leq C \sum_{k, l}\left\|Y_{k, l}\right\|_{L^{\infty}\left(S^{n-1}\right)}\left(\int_{0}^{\infty}\left|J_{\nu(k)}(|x| \rho)\right|^{2} \rho^{1-2 s}\right)^{\frac{1}{2}}\left(\int_{0}^{\infty}\left|g_{k, l}(\rho)\right|^{2} \rho^{2 s+n-1} d \rho\right)^{\frac{1}{2}} \\
& \leq C \sum_{k, l} k^{\frac{n-2}{2}}\left(\frac{\Gamma(\nu(k)+1-s)}{\Gamma(\nu(k)+s)}\right)^{\frac{1}{2}}\left(\int_{0}^{\infty}\left|g_{k, l}(\rho)\right|^{2} \rho^{2 s+n-1} d \rho\right)^{\frac{1}{2}}\left\|Y_{k, l}\right\|_{L^{2}\left(S^{n-1}\right)} .
\end{aligned}
$$

Here we used the inequality that $\left\|Y_{k, l}\right\|_{L^{\infty}} \leq C k^{\frac{n-2}{2}}\left\|Y_{k, l}\right\|_{L^{2}}$ (see for instance [10]). Using the Stirling's formula for gamma function that $\Gamma(t) \approx t^{t-\frac{1}{2}} e^{-(t-1)}$ for large $t$ (for instance, see [1]) and the fact $-\Delta_{\omega} Y_{k, l}=k(k+n-2) Y_{k, l}$, we have from (13)

$$
\begin{aligned}
& |x|^{\frac{n}{2}-s}|u(|x| \omega)| \\
& \leq C \sum_{k} k^{\frac{n-2}{2}} d(k)^{\frac{1}{2}}\left(\frac{\Gamma(\nu(k)+1-s)}{\Gamma(\nu(k)+s)}\right)^{\frac{1}{2}} \\
& \quad \cdot\left(\sum_{1 \leq l \leq d(k)} \int_{0}^{\infty}\left|g_{k, l}(\rho)\right|^{2} \rho^{2 s+n-1} d \rho\left\|Y_{k, l}\right\|_{L^{2}\left(S^{n-1}\right)}^{2}\right)^{\frac{1}{2}} \\
& \leq C\left(\sum_{k} k^{2\left(n-\frac{3}{2}-s-m\right)}\right)^{\frac{1}{2}}\left(\sum_{k, l} k^{2 m} \int_{0}^{\infty}\left|g_{k, l}(\rho)\right|^{2} \rho^{2 s+n-1} d \rho\left\|Y_{k, l}\right\|_{L^{2}\left(S^{n-1}\right)}^{2}\right)^{\frac{1}{2}} \\
& \leq C\left(\sum_{k, l} k^{2 m} \int_{0}^{\infty} \int_{S^{n-1}}\left|\mathcal{F}\left(f_{k, l} Y_{k, l}\right)(\rho \omega)\right|^{2} \rho^{2 s+n-1} d \rho d \omega\right)^{\frac{1}{2}} \\
& \leq C\left(\sum_{k, l} \int_{0}^{\infty} \int_{S^{n-1}}\left|\mathcal{F}\left(\left(1-\Delta_{\omega}\right)^{\frac{m}{2}}\left(f_{k, l} Y_{k, l}\right)\right)(\rho \omega)\right|^{2} \rho^{2 s+n-1} d \rho d \omega\right)^{\frac{1}{2}} \\
& \leq C\|u\|_{\dot{H}_{\omega}^{s, m},}
\end{aligned}
$$


where $\mathcal{F}$ is the Fourier transform. This proves part (1).

For the part (2), if we use the Littlewood-Paley decomposition $\left\{\varphi_{j}\right\}_{j \in \mathbb{Z}}$ as in the proof of Proposition 2, the we have

$$
|x|^{\frac{n-1}{2}} u(|x| \omega)=c_{n} \sum_{j \in \mathbb{Z}} \sum_{k, l}|x|^{\frac{1}{2}} \int_{0}^{\infty} J_{\nu(k)}(|x| \rho) \rho^{\frac{n}{2}} \psi_{j}(\rho) \varphi_{j}(\rho) g_{k, l}(\rho) d \rho Y_{k, l}(\omega),
$$

Since $m>n-3 / 2$, by (12) we deduce that

$$
\begin{aligned}
& |x|^{\frac{n-1}{2}}|u(|x| \omega)| \\
& \leq C \sum_{j \in \mathbb{Z}} \sum_{k, l}\left(\int_{0}^{\infty}\left|\varphi_{j}(\rho) g_{k, l}(\rho)\right|^{2} \rho^{n} d \rho\right)^{\frac{1}{2}}\left\|Y_{k, l}\right\|_{L^{\infty}\left(S^{n-1}\right)} \\
& \leq C \sum_{j \in \mathbb{Z}}\left(\sum_{k} k^{2(n-2-m)}\right)^{\frac{1}{2}}\left(\sum_{k, l} k^{2 m} \int_{0}^{\infty}\left|\varphi_{j}(\rho) g_{k, l}(\rho)\right|^{2} \rho^{n} d \rho\left\|Y_{k, l}\right\|_{L^{2}\left(S^{n-1}\right)}^{2}\right)^{\frac{1}{2}} \\
& \leq C \sum_{j \in \mathbb{Z}} 2^{\frac{j}{2}}\left\|\varphi_{j} \mathcal{F}\left(\left(1-\Delta_{\omega}\right)^{\frac{m}{2}} u\right)\right\|_{L^{2}}=C\|u\|_{\dot{B}_{2,1, \omega}^{\frac{1}{2}}} .
\end{aligned}
$$

To show Corollary 1 we use the fact that $H_{\omega}^{s, m}$ is a Hilbert space. Hence any bounded sequence $\left\{u_{j}\right\}$ in $H_{\omega}^{s, m}$ satisfies $u_{j}(x) \rightarrow 0$ as $|x| \rightarrow \infty$ uniformly and has a subsequence converges to $u$ in $H_{\omega}^{s, m}$ weakly. Let us denote the subsequence by $u_{j_{k}}$.

Now choose a smooth function $\varphi$ supported in the ball of radius $R+1$ and with value 1 in the ball of radius $R$. By the standard argument one can easily show that for each $R$ the mapping $u \mapsto \varphi u$ is compact from $H^{t}$ to $H^{t^{\prime}}$ if $t^{\prime}<t$. By the compactness above and Sobolev embedding we may assume that the sequence $\varphi u_{j_{k}}$ satisfies that for $2 \leq q<\frac{2 n}{n-2 s}$

$$
\left\|\varphi u_{j_{k}}-\varphi u\right\|_{L^{q}} \rightarrow 0 \text { as } k \rightarrow \infty .
$$

Thus we have

$$
\left\|u_{j_{k}}-u\right\|_{L^{p}} \leq\left\|\varphi\left(u_{j_{k}}-u\right)\right\|_{L^{p}}+\left\|(1-\varphi)\left(u_{j_{k}}-u\right)\right\|_{L^{p}} \equiv I_{k}+I I_{k}
$$

with $I_{k} \rightarrow 0$ as $k \rightarrow \infty$ by (16) since $2<p<\frac{2 n}{n-2 s}$. From the uniform convergence that $\left|u_{j_{k}}(x)\right|+|u(x)| \rightarrow 0$ as $|x| \rightarrow \infty$ it follows that

$$
\limsup _{k \rightarrow \infty} I I_{k} \leq \sup _{k}\left\|u_{j_{k}}-u\right\|_{L^{\infty}(|x|>R)}^{\frac{p-2}{p}} \rightarrow 0 \text { as } R \rightarrow \infty .
$$

This proves the compactness of the embedding $H_{\omega}^{s, m} \hookrightarrow L^{p}$.

Since $B_{2,1, \omega}^{\frac{1}{2}, m} \hookrightarrow H_{\omega}^{\frac{1}{2}, m}$, one can adapt the same arguments (compactness of cut-off mapping and uniform convergence at infinity) as above except for weak-* convergence of $u_{j_{k}}$ to $u$ in $B_{2,1, \omega}^{\frac{1}{2}, m}$ for the compactness of embedding $B_{2,1, \omega}^{\frac{1}{2}, m}$ to $L^{p}$. This completes the proof. 


\section{REFERENCES}

[1] G. B. Arfken and H. J. Weber, Mathematical Methods for Physicists, Academic Press, 1995.

[2] T. Cazenave, Semilinear Schrödinger Equations, Courant Lecture Notes in Mathematics. 10, AMS, 2003.

[3] J. Chabrowski, On compact embeddings of radial Sobolev spaces and their applications, Commun. Partial Differential Equations, 17(1992), 941-952.

[4] Y. Ebihara and T.P. Schonbek, On the (non)compactness of the radial Sobolev spaces, Hiroshima Math. J. 16(1986), 665-669.

[5] J. Kato, M. Nakamura and T. Ozawa, A generalization of the weighted Strichartz estimates for wave equations and an application to self-similar solutions, Commun. Pure. Appl. Math. 60(2007), 164-186.

[6] W.-M. Ni, A nonlinear Dirichlet problem on the unit ball and its applications, Indiana Univ. Math. J. 31(1982), 801-807.

[7] W. Rother, Some existence results for the equation $\Delta U+K(x) U^{p}=0$, Commun. Partial Differential Equations. 15(1990), 1461-1473.

[8] W. Sickel and L. Skrzypczak, Radial subspaces of Besov and Lizorkin-Triebel classes: extended Strauss lemma and compactness of embeddings, The Journal of Fourier Analysis and Applications. 6(2000), 639-662.

[9] L. Skrzypczak, Rotation invariant subspaces of Besov and Triebel-Lizorkin space: compactness of embeddings, smoothness and decay of functions, Rev. Mat. Iberoamericana, 18(2002), 267-299.

[10] E. M. Stein and G. Weiss, Introduction to Fourier Analysis on Euclidean Spaces, Princeton Univ. Press, 1971.

[11] W. A. Strauss, Existence of solitary waves in higher dimensions, Commun. Math. Phys. 55(1977), 149-162.

[12] M. E. Taylor, Partial Differential Equations I, Basic Theory, Applied Mathematical Sciences 115, Springer, 1996.

[13] G. Watson, A Treatise on the Theory of Bessel Functions, Reprint of the second (1944) edition. Cambridge Mathematical Library. Cambridge University Press, Cambridge, 1995

Department of Mathematics, POStech, Pohang 790-784, Republic of Korea

E-mail address: changocho@postech.ac.kr

Department of Mathematics, Hokkaido University, Sapporo, 060-0810, Japan

E-mail address: ozawa@math.sci.hokudai.ac.jp 